\title{
Farey series and the Riemann hypothesis
}

\author{
by
}

\section{S. Kanemitsu and M. Yoshimoto (Fukuoka)}

Dedicated to Professor Dr. Katsumi Shiratani

1. Introduction. For any $x \geq 1$, let $F_{x}=F_{[x]}$ denote the sequence of all irreducible fractions with denominator $\leq x$, arranged in increasing order of magnitude:

$$
\begin{aligned}
F_{x}=\left\{\varrho_{\nu}=b_{\nu} / c_{\nu} \mid 0<b_{\nu} \leq c_{\nu} \leq x,\right. & \left.\left(b_{\nu}, c_{\nu}\right)=1\right\} \\
& \left(\varrho_{1}=1 /[x], \varrho_{\Phi(x)-1}=1-1 /[x]\right),
\end{aligned}
$$

called the Farey series (sequence) of order $x$. As points on the unit interval, they are placed symmetrically with respect to the midpoint $1 / 2$.

It is convenient to supplement $\varrho_{0}=0 / 1=b_{0} / c_{0}$ to $F_{x}$ to form $F_{x}^{\prime}$ because it is then easy to construct $F_{x+1}^{\prime}$ from $F_{x}^{\prime}$ by just inserting all mediants $\left(b_{\nu}+b_{\nu+1}\right) /\left(c_{\nu}+c_{\nu+1}\right)$ of successive terms $b_{\nu} / c_{\nu}, b_{\nu+1} / c_{\nu+1}$ in $F_{x}^{\prime}$ between them as long as $c_{\nu}+c_{\nu+1} \leq x+1$. E.g. from $F_{2}^{\prime}=\{0 / 1,1 / 2,1 / 1\}$ we form $F_{3}^{\prime}=\{0 / 1,1 / 3,1 / 2,2 / 3,1 / 1\}$. The number of terms in the Farey series of order $x$ is

$$
\# F_{x}=\Phi(x):=\sum_{n \leq x} \varphi(n)=\frac{3}{\pi^{2}} x^{2}+O(x \log x) .
$$

Here, $\varphi(n)$ stands for the Euler function $\sum_{m \leq n,(m, n)=1} 1$, the number of integers $\leq n$ that are relatively prime to $n$, and is equal to the number of terms in $F_{x}$ whose denominator is $n$.

We put

Hence

$$
\delta_{\nu}=\varrho_{\nu}-\frac{\nu}{\Phi(x)}, \quad \nu=0, \ldots, \Phi(x) .
$$

$$
\delta_{\Phi(x)}=\delta_{0}=0, \delta_{1}=1 /[x]-1 / \Phi(x) \text { etc. }
$$

The celebrated Riemann hypothesis (abbreviated as the RH hereafter) to the effect that all nontrivial zeros of the Riemann zeta-function $\zeta(s)(s=$ $\sigma+i t)$ lie on the critical line $\sigma=1 / 2$ has been the driving force of the developments of modern mathematics in general and of prime number theory 
in particular, and it is formulated in a number of ways. Let $\mu(n)$ denote the Möbius function and let $M(x)$ denote its summatory function:

$$
M(x)=\sum_{n \leq x} \mu(n) .
$$

Then the $\mathrm{RH}$ is equivalent to the estimate

$$
M(x)=O\left(x^{1 / 2+\varepsilon}\right) \quad \text { for every } \varepsilon>0 .
$$

In the sequel $\varepsilon$ will always be used in this context and the proviso "for every $\varepsilon>0$ " will not be repeated. Another form which will be used in Section 5 is in terms of Chebyshev's function

$$
\psi(x)=\sum_{n \leq x} \Lambda(n)=\sum_{p^{m} \leq x} \log p,
$$

with $\Lambda(n)$ denoting the von Mangoldt function

$$
\Lambda(n)= \begin{cases}\log p, & n=p^{m} \\ 0, & \text { otherwise. }\end{cases}
$$

The $\mathrm{RH}$ is equivalent to the estimate

$$
\psi(x)=x+O\left(x^{1 / 2+\varepsilon}\right) .
$$

There are many other conditions equivalent to the RH (cf. Kano [8]). It has long been known that there is a close connection between the distribution of Farey points and the RH, notably in view of the classical Franel identity (Theorem 3 below) obtained first by Franel [3] and expounded then by Landau [11], [12]. More recently there have appeared some other types of results revealing the connection between Farey points and the RH, e.g. power moments results in Corollary 2 to Proposition 1 due to Kopriva [9], [10], Mikolás [14], [15] and Zulauf [21], which are, however, merely very special cases of Mikolás's general theorem for polynomials of degree $\leq 3$ ([14], Theorem 5). Huxley [6] has generalized Franel's theorem to the case of Dirichlet $L$-functions and Fujii [4], [5] has obtained another equivalent condition to the $\mathrm{RH}$ in terms of Farey series. All the papers mentioned above are concerned with conditions equivalent to the $\mathrm{RH}$ in terms of Farey series.

On the other hand, Codecà [1] and Codecà and Perelli [2] have considered the problem of estimating the error $E_{f}(x)$ to be defined below under the (weaker) RH.

That the Farey sequence is uniformly distributed mod 1 is easily seen in Mikolás [14], p. 99 as follows. Arrange all Farey fractions in the order of appearance in $F_{x}$ to form the Farey sequence $\left\{x_{n}\right\}$. Suppose the $n$th term lies in $F_{x+1}-F_{x}$. Then $n=\Phi(x)+O(x)$. On the other hand, the number $A_{n}(a, b)$ of terms among $x_{1}, \ldots, x_{n}$ that lie in a given subinterval 
$[a, b] \subset[0,1]$ is, by Corollary to Lemma 3 ,

$$
A_{n}(a, b)=(b-a) \Phi(x)+O(x \log x) \sim(b-a) n, \quad x \rightarrow \infty .
$$

Hence, by Weyl's criterion, for every Riemann integrable function $f$ on $(0,1)$, we have

$$
\lim _{x \rightarrow \infty} \frac{1}{\Phi(x)} \sum_{\nu=1}^{\Phi(x)} f\left(\varrho_{\nu}\right)=\int_{0}^{1} f(t) d t=: A
$$

so that the problem of estimating the error

$$
E_{f}(x):=\sum_{\nu=1}^{\Phi(x)} f\left(\varrho_{\nu}\right)-A \Phi(x)
$$

or more generally, with $0<\xi \leq 1$, estimating the short interval error

$$
E_{f}(\xi, x):=\sum_{\varrho_{\nu} \leq \xi} f\left(\varrho_{\nu}\right)-\Phi(x) \int_{0}^{\xi} f(t) d t \quad\left(E_{f}(1, x)=E_{f}(x)\right)
$$

would be interesting, and it would be all the more so if we could formulate the RH in terms of $E_{f}(x)$ for a wide class of functions $f$ because then there might be some hope to find means independent of the RH to estimate $E_{f}(x)$ directly, thereby providing a possible approach to the $\mathrm{RH}$.

Our main objective is to find equivalent conditions to the $\mathrm{RH}$ for a rather wide class of functions, i.e. Kubert functions (for these cf. $\S 5$ ), as well as some unexpected results for short intervals. From the point of view of the parity of Kubert functions, the meaning of formulas (3) and (i) and (ii) in Corollary 2 to Proposition 1 is rendered very visible. Indeed, formula (3) is the sum of the odd part $f^{\text {odd }}(x):=\frac{1}{2}\{f(x)-f(1-x)\}$ of the logarithmic function $\log \left(1-e^{2 \pi i x}\right)$ over the whole range (cf. the formula in (i1) in Corollary to Proposition 3), which is therefore trivial on account of the general formula (cf. Mikolás [14], p. 108 in this regard)

$$
2 \sum_{\nu=1}^{\Phi(x)-1} f^{\text {odd }}\left(\varrho_{\nu}\right)=\sum_{\nu=1}^{\Phi(x)-1}\left\{f\left(\varrho_{\nu}\right)-f\left(1-\varrho_{\nu}\right)\right\}=0
$$

while for the even part of $f\left(f^{\text {even }}(x):=\frac{1}{2}\{f(x)+f(1-x)\}\right)$

$$
2 \sum_{\varrho_{\nu} \leq 1 / 2} f^{\text {even }}\left(\varrho_{\nu}\right)=\sum_{\varrho_{\nu} \leq 1 / 2}\left\{f\left(\varrho_{\nu}\right)+f\left(1-\varrho_{\nu}\right)\right\}=\sum_{\nu=1}^{\Phi(x)-1} f\left(\varrho_{\nu}\right)+f(1 / 2) .
$$

Hence, for even $f$, the sum over the whole interval amounts to the sum over $\frac{1}{2}$-interval $\left(\varrho_{\nu} \leq 1 / 2\right)$, whilst for odd $f$, only half (or shorter) interval results are significant, in which regard Zulauf's $\frac{1}{2}$-interval $\left(\varrho_{\nu} \leq 1 / 2\right)$ result [21] in Corollary 1 to Theorem 1 is noteworthy, but somehow natural because it 
may be that the half-interval still represents the distribution of Farey points on the whole unit interval.

However, as the first aim in this paper we proceed still further and establish $\frac{1}{3}-, \frac{1}{4}$-results (Corollaries 2 and 3 to Theorem 1), which seem rather remarkable in that in shorter intervals the Farey points still have some relevance to the RH.

The second objective is to obtain conditions equivalent to the $\mathrm{RH}$ for Kubert functions. Thus, we significantly extend the class of functions in terms of which we can formulate conditions equivalent to the RH compared to that considered by Mikolás [14]. From this standpoint, formula (i) in Corollary 2 to Proposition 1 is nothing but the first formula (even part of $\left.\ell_{2}(x)\right)$ in Corollary 2 to Theorem 2. In fact, we have

$$
\sum_{\nu=1}^{\Phi(x)} \bar{B}_{2}\left(\varrho_{\nu}\right)=\sum_{\nu=1}^{\Phi(x)}\left(\varrho_{\nu}^{2}-\frac{1}{3}\right)-B_{1}
$$

Likewise, formula (ii) in Corollary 2 to Proposition 1 readily follows from Corollary 1(i) to Proposition 3:

$$
\begin{aligned}
\sum_{\nu=1}^{\Phi(x)}\left(\varrho_{\nu}^{3}-\frac{1}{4}\right) & =\sum_{\nu=1}^{\Phi(x)} B_{3}\left(\varrho_{\nu}\right)+\frac{3}{2} \sum_{\nu=1}^{\Phi(x)} B_{2}\left(\varrho_{\nu}\right)+\sum_{\nu=1}^{\Phi(x)} B_{1}\left(\varrho_{\nu}\right) \\
& =\frac{3}{2} B_{2} S(x)+\frac{1}{2} .
\end{aligned}
$$

Finally, we will show that the well-known Franel formula has its origin in the three-term relation for the generalized Dedekind sums.

Other conditions equivalent to the RH in the spirit of Mikolás [15] and Mikolás-Sato [16] have been obtained by the second-named author and are being prepared for publication.

We summarize our main contributions:

(i) Each of the estimates

$$
\sum_{\varrho_{\nu} \leq 1 / 3}\left(\varrho_{\nu}-\frac{h(1 / 3)}{2 \Phi(x)}\right)=O\left(x^{1 / 2+\varepsilon}\right), \quad \sum_{\varrho_{\nu} \leq 1 / 4}\left(\varrho_{\nu}-\frac{h(1 / 4)}{2 \Phi(x)}\right)=O\left(x^{1 / 2+\varepsilon}\right)
$$

is equivalent to the $\mathrm{RH}$.

(ii) For $\sigma \geq 3 / 2$ and any extended even Kubert function $f \in \mathcal{K}_{s}$, the estimate

$$
\sum_{\nu=1}^{\Phi(x)} f\left(\varrho_{\nu}\right)=O\left(x^{1 / 2+\varepsilon}\right)
$$

is equivalent to the RH. 
(iii) Franel's formula

$$
\sum_{\nu=1}^{\Phi(x)} \delta_{\nu}^{2}=\frac{1}{12 \Phi(x)}\left(\sum_{m, n \leq x} M\left(\frac{x}{m}\right) M\left(\frac{x}{n}\right) \frac{(m, n)^{2}}{m n}-1\right)
$$

is essentially a consequence of the three-term relation for the generalized Dedekind sums.

Acknowledgements. The authors would like to thank Prof. Dr. R. Sczech of Kyushu University and Rutgers University for enlightening discussions about Dedekind sums.

\section{Preliminaries}

Lemma 1 (Ishibashi and Kanemitsu [7], Lemma 3). For any $u \in \mathbb{N} \cup\{0\}$ and any $x \rightarrow \infty$, let $L_{u}(x):=\sum_{n \leq x} n^{u}$. Then

$$
L_{u}(x)=\frac{1}{u+1} \sum_{r=0}^{u+1}(-1)^{r}\left(\begin{array}{c}
u+1 \\
r
\end{array}\right) \bar{B}_{r}(x) x^{u+1-r}+\zeta(-u),
$$

where $\bar{B}_{r}(x)=B_{r}(\{x\})$ with $B_{r}(x)$ and $\{x\}$ denoting the rth Bernoulli polynomial and the fractional part of $x$, respectively.

Definition 1. For any $s=\sigma+i t \in \mathbb{C}$ define

$$
S_{s}(x):=\sum_{n \leq x}\left(\mu * N^{-s}\right)(n)=\sum_{n \leq x} M\left(\frac{x}{n}\right) \frac{1}{n^{s}},
$$

where $N^{-s}(n)=n^{-s}$ and $*$ means the Dirichlet convolution:

$$
(f * g)(n)=\sum_{d \mid n} f(d) g\left(\frac{n}{d}\right)=\sum_{d \delta \leq n} f(d) g(\delta) .
$$

We make good use of the particular sum

$$
S(x):=S_{1}(x)=\sum_{n \leq x} M\left(\frac{x}{n}\right) \frac{1}{n}
$$

subsequently.

Lemma 2 (Mikolás [14], Lemma 7). For each $s$ with $\operatorname{Re} s \geq 1 / 2$, the estimate $S_{s}(x)=O\left(x^{1 / 2+\varepsilon}\right)$ is equivalent to the $R H$.

Pr o of. For $\operatorname{Re} z>1$, the generating Dirichlet series for $\mu$ and $N^{-s}$ are

$$
\sum_{n=1}^{\infty} \mu(n) n^{-z}=\zeta(z)^{-1}, \quad \sum_{n=1}^{\infty} N^{-s}(n) n^{-z}=\zeta(z+s)
$$


respectively. Hence

$$
\sum_{n=1}^{\infty}\left(\mu * N^{-s}\right)(n) n^{-z}=\zeta(z+s) \zeta(z)^{-1} .
$$

By partial summation we have

$$
\frac{\zeta(z+s)}{\zeta(z)}=z \int_{1}^{\infty} \frac{S_{s}(x)}{x^{z+1}} d x .
$$

If $S_{s}(x)=O\left(x^{1 / 2+\varepsilon}\right)$, then the integral is absolutely and uniformly convergent for $\operatorname{Re} z>1 / 2$, and so it represents an analytic function for $\operatorname{Re} z>1 / 2$. And so is $\zeta(z+s) / \zeta(z)$, implying that $\zeta(z)$ has no zeros for $\operatorname{Re} z>1 / 2$, which amounts to the RH.

From the RH in the form of (1) we immediately deduce the estimate for $S_{s}(x)$, thereby completing the proof.

LEMMA 3 (Generalization of Lemma 4 of Mikolás [14] and of Lemma 2 of Zulauf [21]). Let $0<\xi \leq 1$ and let $f(t)$ be defined at the points $m / n((m, n)$ $=1, m \leq n \leq x)$. Then

$$
h(\xi, f):=\sum_{\varrho_{\nu} \leq \xi} f\left(\varrho_{\nu}\right)=\sum_{n \leq x}\left(\mu * V_{\xi}\right)(n)=\sum_{n \leq x} M\left(\frac{x}{n}\right) V_{\xi}(n),
$$

where $V_{\xi}(n):=\sum_{k \leq n \xi} f(k / n)$.

P r o o f. The proof runs along similar lines to those of Mikolás and Zulauf:

$$
\begin{aligned}
h(\xi, f) & =\sum_{n \leq x} \sum_{\substack{m / n \leq \xi \\
(m, n)=1}} f\left(\frac{m}{n}\right)=\sum_{n \leq x} \sum_{m / n \leq \xi} f\left(\frac{m}{n}\right) \sum_{d \mid(m, n)} \mu(d) \\
& =\sum_{n \leq x} \sum_{d \mid n} \mu(d) \sum_{\substack{m / n \leq \xi \\
d \mid m}} f\left(\frac{m}{n}\right) \\
& =\sum_{d \delta \leq x} \mu(d) \sum_{m^{\prime} / \delta \leq \xi} f\left(\frac{m^{\prime}}{\delta}\right) \\
& \left(\text { on writing } n=d \delta, m=d m^{\prime} \text { in the innermost sum }\right) \\
& =\sum_{d \delta \leq x} \mu(n) V_{\xi}(\delta)=\sum_{n \leq x}\left(\mu * V_{\xi}\right)(n),
\end{aligned}
$$

which completes the proof.

Corollary.

$$
h(\xi):=\sum_{\varrho_{\nu} \leq \xi} 1=\sum_{n \leq x} M\left(\frac{x}{n}\right)[n \xi] .
$$


We note the following identities which are immediate consequences of Lemma 3 and will be utilized very often without further mentioning:

$$
S_{-1}(x)=\sum_{n \leq x} M\left(\frac{x}{n}\right) n=\Phi(x), \quad S_{0}(x)=\sum_{n \leq x} M\left(\frac{x}{n}\right)=1 .
$$

\section{Power moments}

Proposition 1. For every $k \in \mathbb{N} \cup\{0\}$ and $0<\xi \leq 1$,

$$
\begin{aligned}
\sum_{\varrho_{\nu} \leq \xi} \varrho_{\nu}^{k}= & \frac{1}{k+1} \xi^{k+1} \Phi(x) \\
& +\frac{1}{k+1} \sum_{r=1}^{k}(-1)^{r}\left(\begin{array}{c}
k+1 \\
r
\end{array}\right) \xi^{k+1-r} \sum_{n \leq x} M\left(\frac{x}{n}\right) \frac{1}{n^{r-1}} \bar{B}_{r}(n \xi) \\
& +\frac{(-1)^{k+1}}{k+1} \sum_{n \leq x} M\left(\frac{x}{n}\right) \frac{1}{n^{k}} \bar{B}_{k+1}(n \xi)+\zeta(-k) S_{k}(x) .
\end{aligned}
$$

In particular,

$$
h(\xi)=\sum_{\varrho_{\nu} \leq \xi} 1=\xi \Phi(x)-\sum_{n \leq x} M\left(\frac{x}{n}\right)\{n \xi\} .
$$

Proof. Since, by Lemma 3,

$$
\sum_{\varrho_{\nu} \leq \xi} \varrho_{\nu}^{k}=\sum_{n \leq x} M\left(\frac{x}{n}\right) \sum_{m \leq n \xi}\left(\frac{m}{n}\right)^{k}=\sum_{n \leq x} M\left(\frac{x}{n}\right) \frac{1}{n^{k}} L_{k}(n \xi),
$$

the assertion follows on substituting for $L_{k}(n \xi)$ from Lemma 1 and separating the terms with $r=0$ and $r=k+1$.

Remark 1. Codecà [1] proves that for irrational $\xi$ of infinite type, the infimum of $\xi$ such that $E_{1}(\xi, x):=h(\xi)-\xi \Phi(x)=O\left(x^{\beta}\right)$ is equal to 1 and that if $\xi \neq 1 / 2$ is rational, then under the $\mathrm{RH}$ and the Lindelöf hypothesis for some Dirichlet $L$-functions, one has $E_{1}(\xi, x)=O\left(x^{1 / 2+\varepsilon}\right)$.

Corollary 1. For every $k \in \mathbb{N} \cup\{0\}$,

$$
\sum_{\nu=1}^{\Phi(x)}\left(\varrho_{\nu}^{k}-\frac{1}{k+1}\right)=\frac{1}{k+1} \sum_{r=1}^{k}(-1)^{r}\left(\begin{array}{c}
k+1 \\
r
\end{array}\right) B_{r} S_{r-1}(x) .
$$

This is because the $3 \mathrm{rd}$ and the 4 th terms cancel each other in view of

$$
(-1)^{k+1} \frac{B_{k}}{k+1}+\zeta(-k)=0 .
$$


In particular, for $k=0$, this just says $\sum_{\nu=1}^{\Phi(x)} 1=\Phi(x)$, and for $k=1$,

$$
\sum_{\nu=1}^{\Phi(x)}\left(\varrho_{\nu}-\frac{1}{2}\right)=\frac{1}{2} .
$$

Corollary 2 (Kopriva, Mikolás, Zulauf). Each of the following assertions is equivalent to the Riemann hypothesis:

$$
\begin{aligned}
& \sum_{\nu=1}^{\Phi(x)}\left(\varrho_{\nu}^{2}-\frac{1}{3}\right)=O\left(x^{1 / 2+\varepsilon}\right), \\
& \sum_{\nu=1}^{\Phi(x)}\left(\varrho_{\nu}^{3}-\frac{1}{4}\right)=O\left(x^{1 / 2+\varepsilon}\right) .
\end{aligned}
$$

Pr o of. From Corollary 1 to Proposition 1 we immediately deduce

$$
\sum_{\nu=1}^{\Phi(x)}\left(\varrho_{\nu}^{2}-\frac{1}{3}\right)=\frac{1}{2}+\frac{1}{6} S(x),
$$

and

$$
\sum_{\nu=1}^{\Phi(x)}\left(\varrho_{\nu}^{3}-\frac{1}{4}\right)=\frac{1}{2}+\frac{1}{4} S(x)
$$

which prove respectively (i) and (ii) on the basis of Lemma 2 .

\section{Short interval results}

Proposition 2. For any $0<\xi \leq 1$ we have

$$
\begin{aligned}
\sum_{\varrho_{\nu} \leq \xi}\left(\varrho_{\nu}-\frac{h(\xi)}{2 \Phi(x)}\right)= & \frac{1}{2} \Phi(x) \xi^{2}-\frac{h(\xi)^{2}}{2 \Phi(x)}-\xi \sum_{n \leq x} M\left(\frac{x}{n}\right) \bar{B}_{1}(n \xi) \\
& +\frac{1}{2} \sum_{n \leq x} M\left(\frac{x}{n}\right) \frac{1}{n} \bar{B}_{2}(n \xi)-\frac{1}{12} S(x),
\end{aligned}
$$

where $h(\xi)=h(\xi, x)$ counts the number of $\varrho_{\nu}$ 's $\leq \xi$ as in Corollary to Lemma 3.

Pr o of. This is nothing but Proposition 1 with $k=1$, which reads

$$
\begin{aligned}
\sum_{\varrho_{\nu} \leq \xi} \varrho_{\nu}= & \frac{1}{2} \Phi(x) \xi^{2}-\xi \sum_{n \leq x} M\left(\frac{x}{n}\right) \bar{B}_{1}(n \xi) \\
& +\frac{1}{2} \sum_{n \leq x} M\left(\frac{x}{n}\right) \frac{1}{n} \bar{B}_{2}(n \xi)-\frac{1}{12} S(x) .
\end{aligned}
$$

Subtracting $\sum_{\varrho_{\nu} \leq \xi} h(\xi) /(2 \Phi(x))$ from the left-hand side has the effect of placing the second term on the right-hand side of the above formula. 
Lemma 4. (i) Define the odd part of $S_{s}(x)$ by

$$
S_{s}^{\text {odd }}(x)=\sum_{\substack{n \leq x \\ 2 \nmid n}} M\left(\frac{x}{n}\right) \frac{1}{n^{s}},
$$

which we abbreviate as $\sum_{2 \nmid n} M\left(\frac{x}{n}\right) \frac{1}{n^{s}}$. Then

$$
S_{s}^{\text {odd }}(x)=S_{s}(x)-\frac{1}{2^{s}} S_{s}\left(\frac{x}{2}\right) .
$$

(ii) (Telescoping series) For any $0<c \leq 1$ and $1<N \in \mathbb{N}$ we have

$$
\begin{gathered}
S_{s}(x)=\sum_{r=0}^{\infty}(\mp c)^{r}\left(S_{s}\left(\frac{x}{N^{r}}\right) \pm c S_{s}\left(\frac{x}{N^{r+1}}\right)\right) \\
S_{s}^{\text {nondiv }}(x)=\sum_{r=0}^{\infty}(\mp c)^{r}\left(S_{s}^{\text {nondiv }}\left(\frac{x}{N^{r}}\right) \pm c S_{s}^{\text {nondiv }}\left(\frac{x}{N^{r+1}}\right)\right),
\end{gathered}
$$

so that for $\beta>0$ any of the estimates of the form

$$
\begin{gathered}
S_{s}^{\text {nondiv }}(x):=S_{s}(x)-\frac{1}{N^{s}} S_{s}\left(\frac{x}{N}\right)=O\left(x^{\beta}\right), \\
S_{s}^{\text {nondiv }}(x) \pm c S_{s}^{\text {nondiv }}\left(\frac{x}{N}\right)=O\left(x^{\beta}\right)
\end{gathered}
$$

is equivalent to the estimate $S_{s}(x)=O\left(x^{\beta}\right)$.

(iii) Similar results hold for Chebyshev's function $\psi$ : The estimate

$$
\psi^{\text {odd }}(x):=\psi(x)-\frac{1}{2} \psi\left(\frac{x}{2}\right)=O\left(x^{\beta}\right)
$$

is equivalent to the estimate $\psi(x)=O\left(x^{\beta}\right)$.

Proof. (i) follows on observing, after dividing $S_{s}(x)$ into the even and odd parts, that the even part is $\frac{1}{2} S_{s}(x / 2)$.

(ii) The first formula is clear. Notice that it is a finite sum with $[\log x / \log N]=O(\log x)$ terms. This last remark shows that the estimate $S_{s}(x) \pm c S_{s}(x / N)=O\left(x^{\beta}\right)$ implies the estimate $S_{s}(x)=O\left(x^{\beta}\right)$ since there are $O(\log x)$ terms of order $O\left(x^{\beta}\right)$, the reverse implication being trivial. Similarly for $S_{s}^{\text {odd }}(x)$. The proof of (iii) is similar to that of (ii).

TheOREM 1. We have

$$
\sum_{\varrho_{\nu} \leq \xi}\left(\varrho_{\nu}-\frac{h(\xi)}{2 \Phi(x)}\right)=\frac{1}{2} T(\xi, x)+O(1),
$$

where

$$
T(\xi, x)=\sum_{n \leq x} M\left(\frac{x}{n}\right) \frac{1}{n}\{n \xi\}(\{n \xi\}-1) .
$$


Proof. From Corollary to Lemma 3 we have, on using Lemma 3 of Niederreiter [18],

$$
\begin{aligned}
\frac{h(\xi)^{2}}{2 \Phi(x)}= & \frac{1}{2 \Phi(x)}\left(\xi^{2} \Phi(x)^{2}-2 \xi \Phi(x) \sum_{n \leq x} M\left(\frac{x}{n}\right)\left(\bar{B}_{1}(n \xi)+\frac{1}{2}\right)\right) \\
& +O\left(\frac{1}{x^{2}}\left(\sum_{n \leq x}\left|M\left(\frac{x}{n}\right)\right|\right)^{2}\right) \\
= & \frac{1}{2} \Phi(x) \xi^{2}-\xi \sum_{n \leq x} M\left(\frac{x}{n}\right) \bar{B}_{1}(n \xi)+O(1) .
\end{aligned}
$$

Substituting this in the formula of Proposition 2 and putting together the last two terms, we get the assertion.

Remark 2. (i) Regarding the error term $O(1)$ in the formula of Theorem 1 we note the following. As a function of $\xi$ it can be explicitly written as

$$
\frac{\xi}{2}-\frac{1}{2 \Phi(x)} E_{1}(\xi, x)^{2}
$$

where $E_{1}(\xi, x)=h(\xi)-\xi \Phi(x)$ is the short interval error stated in Section 1 (with $f(t) \equiv 1$ ). Theorem 2 of Codecà [1] asserts that under the RH and the Lindelöf hypothesis for certain Dirichlet $L$-functions, one has

$$
E_{1}(\xi, x)=O\left(x^{1 / 2+\varepsilon}\right) \text { or } O(1) \quad \text { according as } \xi \neq 1 / 2 \text { or } \xi=1 / 2 .
$$

This seems to suggest that the error term cannot probably be replaced by $o(1)$.

(ii) From the identity

$$
\sum_{\varrho_{\nu} \leq \xi} \nu=\sum_{\nu=1}^{h(\xi)} \nu=\frac{h(\xi)(h(\xi)+1)}{2}=\frac{1}{2} h(\xi)+\sum_{\varrho_{\nu} \leq \xi} \frac{h(\xi)}{2},
$$

it follows that

$$
\begin{aligned}
\sum_{\varrho_{\nu} \leq \xi} \delta_{\nu} & :=\sum_{\varrho_{\nu} \leq \xi}\left(\varrho_{\nu}-\frac{\nu}{\Phi(x)}\right)=\sum_{\varrho_{\nu} \leq \xi}\left(\varrho_{\nu}-\frac{h(\xi)}{2 \Phi(x)}\right)-\frac{h(\xi)}{2 \Phi(x)} \\
& =\sum_{\varrho_{\nu} \leq \xi}\left(\varrho_{\nu}-\frac{h(\xi)}{2 \Phi(x)}\right)+O(1) .
\end{aligned}
$$

Hence the statement

$$
\max _{0<\xi \leq 1}\left|\sum_{\varrho_{\nu} \leq \xi}\left(\varrho_{\nu}-\frac{h(\xi)}{2 \Phi(x)}\right)\right|=O\left(x^{1 / 2+\varepsilon}\right)
$$


is, via Landau's theorem to the effect that

$$
\max _{0<\xi \leq 1}\left|\sum_{\varrho_{\nu} \leq \xi} \delta_{\nu}\right|=O\left(x^{1 / 2+\varepsilon}\right),
$$

equivalent to the $\mathrm{RH}$.

However, we can give the following stronger statements (i.e. with weaker conditions) for short intervals.

Corollary 1 (Zulauf). We have

$$
\begin{gathered}
\sum_{\varrho_{\nu} \leq 1 / 2}\left(\varrho_{\nu}-\frac{h(1 / 2)}{2 \Phi(x)}\right)=-\frac{1}{8} S^{\text {odd }}(x)+O(1), \\
S^{\text {odd }}(x)=S_{1}^{\text {odd }}(x)=S(x)-\frac{1}{2} S\left(\frac{x}{2}\right)
\end{gathered}
$$

and hence the estimate

$$
\sum_{\varrho_{\nu} \leq 1 / 2}\left(\varrho_{\nu}-\frac{1}{4}\right)=O\left(x^{1 / 2+\varepsilon}\right)
$$

is equivalent to the Riemann hypothesis.

Pro of. It suffices to compute the sum

$$
T\left(\frac{1}{2}, x\right)=\sum_{n \leq x} M\left(\frac{x}{n}\right) \frac{1}{n}\left\{\frac{n}{2}\right\}\left(\left\{\frac{n}{2}\right\}-1\right),
$$

which is easily done: Divide it into even and odd parts, and then notice that the even part is 0 , while the odd part is $-\frac{1}{4} S_{1}^{\text {odd }}(x)$, which is given in Lemma 4, thereby proving the first formula. Since in $F_{x}^{\prime}$ the terms $\varrho_{\nu}$ and $1-\varrho_{\nu}$ are placed symmetrically with regard to $1 / 2$ and they are $\Phi(x)$ in number, we derive that $h(1 / 2)=\frac{1}{2} \Phi(x)$, which completes the proof.

Remark 3. The formula of Theorem 1 seems to be the proper formulation of a condition equivalent to the $\mathrm{RH}$ in terms of a short interval sum of $\varrho_{\nu}$ 's themselves since it gives not only a conceptually much simpler proof of Zulauf's theorem but also gives another short interval result:

Corollary 2. We have

$$
\sum_{\varrho_{\nu} \leq 1 / 3}\left(\varrho_{\nu}-\frac{h(1 / 3)}{2 \Phi(x)}\right)=-\frac{2}{9}\left(S(x)-\frac{1}{3} S\left(\frac{x}{3}\right)\right)+O(1),
$$

so that the estimate

$$
\sum_{\varrho_{\nu} \leq 1 / 3}\left(\varrho_{\nu}-\frac{h(1 / 3)}{2 \Phi(x)}\right)=O\left(x^{1 / 2+\varepsilon}\right)
$$

is equivalent to the $R H$. 
Proof. To prove the first formula, divide the sum

$$
T\left(\frac{1}{3}, x\right)=\sum_{n \leq x} M\left(\frac{x}{n}\right) \frac{1}{n}\left\{\frac{n}{3}\right\}\left(\left\{\frac{n}{3}\right\}-1\right)
$$

into three parts according to the residue classes modulo 3 to obtain

$$
T\left(\frac{1}{3}, x\right)=-\frac{2}{9} \sum_{n \neq 0(\bmod 3)} M\left(\frac{x}{n}\right) \frac{1}{n} .
$$

Applying the same argument to $S(x)$ we find that

$$
\sum_{n \neq 0(\bmod 3)} M\left(\frac{x}{n}\right) \frac{1}{n}=S(x)-\frac{1}{3} S\left(\frac{x}{3}\right)
$$

which is an analogue of Lemma 3 in the case of modulo 3 . This proves the first formula.

The second formula follows from the first and Lemma 3(ii) with $N=3$, $c=1$.

Corollary 3. We have

$$
\sum_{\varrho_{\nu} \leq 1 / 4}\left(\varrho_{\nu}-\frac{h(1 / 4)}{2 \Phi(x)}\right)=-\frac{3}{16}\left(S^{\text {odd }}(x)+\frac{2}{3} S^{\text {odd }}\left(\frac{x}{2}\right)\right)+O(1),
$$

so that the estimate

$$
\sum_{\varrho_{\nu} \leq 1 / 4}\left(\varrho_{\nu}-\frac{h(1 / 4)}{2 \Phi(x)}\right)=O\left(x^{1 / 2+\varepsilon}\right)
$$

is equivalent to the $R H$.

Pr o of. Dividing into residue classes $\bmod 4$, we get

$$
\begin{aligned}
T\left(\frac{1}{4}, x\right)= & -\frac{3}{16}\left(\sum_{n \equiv 1(\bmod 4)}+\sum_{n \equiv 3(\bmod 4)}\right) \\
& -\frac{1}{4} \sum_{2 m-1 \leq x / 2} M\left(\frac{x / 2}{2 m-1}\right) \frac{1}{2(2 m-1)} \\
= & -\frac{3}{16} S^{\text {odd }}(x)-\frac{1}{8} S^{\text {odd }}\left(\frac{x}{2}\right)
\end{aligned}
$$

implying the first formula.

The second formula follows if we apply Lemma 4(ii) with $N=2, c=2 / 3$.

Re mark 4. In Corollaries 2 and 3, the behavior of $h(\xi)$ is more delicate than in Corollary 1. 
(i) We have on the one hand,

$$
\begin{aligned}
h\left(\frac{1}{3}\right) & =\frac{1}{3} \Phi(x)-\sum_{n \equiv 1,2(\bmod 3)} M\left(\frac{x}{n}\right)\left\{\frac{n}{3}\right\} \\
& =\frac{1}{3} \Phi(x)-\frac{1}{3} \sum_{n \equiv 1(\bmod 3)} M\left(\frac{x}{n}\right)-\frac{2}{3} \sum_{n \equiv 2(\bmod 3)} M\left(\frac{x}{n}\right),
\end{aligned}
$$

while on the other hand,

$$
1=S_{0}(x)=S_{0}\left(\frac{x}{3}\right)+\sum_{n \equiv 1,2(\bmod 3)} M\left(\frac{x}{n}\right)=1+\sum_{n \equiv 1,2(\bmod 3)} M\left(\frac{x}{n}\right),
$$

so that

This implies that

$$
\sum_{n \equiv 1,2(\bmod 3)} M\left(\frac{x}{n}\right)=0
$$

$$
h\left(\frac{1}{3}\right)=\frac{1}{3} \Phi(x)-\frac{1}{3} \sum_{n \equiv 2(\bmod 3)} M\left(\frac{x}{n}\right) .
$$

We do not know how to treat the second term, and this leaves a subtle ingredient in that if we just subtract the number $1 / 3$, we could not get any result.

(ii) Similarly,

$$
\begin{aligned}
h\left(\frac{1}{4}\right)= & \frac{1}{4} \Phi(x)-\frac{1}{2} S_{0}^{\text {odd }}\left(\frac{x}{2}\right)-\frac{1}{4} \sum_{n \equiv 1(\bmod 4)} M\left(\frac{x}{n}\right) \\
& -\frac{3}{4} \sum_{n \equiv 3(\bmod 4)} M\left(\frac{x}{n}\right),
\end{aligned}
$$

and

$$
\sum_{n \equiv 1,3(\bmod 4)} M\left(\frac{x}{n}\right)=0,
$$

since $S_{0}^{\text {odd }}(x / 2)=0$. Thus it follows that

$$
h\left(\frac{1}{4}\right)=\frac{1}{4} \Phi(x)-\frac{1}{2} \sum_{n \equiv 3(\bmod 4)} M\left(\frac{x}{n}\right) .
$$

\section{Kubert functions}

Definition 2. For a fixed complex number $s$, let $\mathcal{K}_{s}$ denote the complex vector space of all continuous functions $f:(0,1) \rightarrow \mathbb{C}$ satisfying the Kubert 
identity

$$
f(x)=m^{s-1} \sum_{k=0}^{m-1} f\left(\frac{x+k}{m}\right)
$$

for every $m \in \mathbb{N}$ and every $x \in(0,1)$.

Lemma 5 (Milnor [17], Theorem 1). We have $\operatorname{dim}_{\mathbb{C}} \mathcal{K}_{s}=2$ and $\mathcal{K}_{s}$ is spanned by one even element $(f(1-x)=f(x))$ and one odd element $(f(1-x)=-f(x))$. For $s \neq-1,-2, \ldots$ the space $\mathcal{K}_{s}$ is spanned by two linearly independent functions $\ell_{s}(x)$ and $\ell_{s}(1-x)$, the polylogarithms of complex exponential argument $\ell_{s}(x)=\sum_{n=1}^{\infty} e^{2 \pi i n x} / n^{s}, \sigma>1, x \in \mathbb{R}$ (also for $\operatorname{Im} x>0, s \in \mathbb{C})$, while for $s \neq 0,1,2, \ldots, \mathcal{K}_{s}$ is spanned by two linearly independent functions $\zeta_{1-s}(x)$ and $\zeta_{1-s}(1-x)$, with the Hurwitz zeta-function $\zeta_{s}(x)=\sum_{n=0}^{\infty} 1 /(n+x)^{s}(\sigma>1)$.

Lemma 6 (Milnor [17], Lemma 7). If a function $f:(0,1) \rightarrow \mathbb{C}$ satisfies the Kubert identities $\left(*_{s}\right)$ with $s \neq 1$, then by choosing $f(0)$ appropriately, it extends uniquely to a function $f: \mathbb{R} / \mathbb{Z} \rightarrow \mathbb{C}$ satisfying $\left(*_{s}\right)$. In particular, if $f \in \mathcal{K}_{s}$ with $\sigma>1$, then the extension is also continuous; for $f(x)=\ell_{s}(x)(\sigma>1)$ the appropriate choice is $f(0)=\zeta(s)$, while for $f(x)=\zeta_{1-s}(x)(\sigma<0)$, it is $f(0)=\zeta(1-s)$.

For the polylogarithm function see also Lewin [13] and Yamamoto [20].

Proposition 3. (i) For every $n \in \mathbb{N}$ and every $f: \mathbb{R} / \mathbb{Z} \rightarrow \mathbb{C}$ satisfying $\left(*_{s}\right)$ with $s \neq 1$, we have

$$
\sum_{\nu=1}^{\Phi(x)} f\left(n \varrho_{\nu}\right)=f(0) \sum_{m \leq x} M\left(\frac{x}{m}\right) \frac{(m, n)^{s}}{m^{s-1}} .
$$

(ii) For the same $f$ as in (i),

$$
\begin{aligned}
f(0) \sum_{m, n \leq x} M\left(\frac{x}{m}\right) M\left(\frac{x}{n}\right) \frac{(m, n)^{s}}{(m n)^{s-1}} & =\sum_{n \leq x} M\left(\frac{x}{n}\right) \frac{1}{n^{s}} \sum_{\nu=1}^{\Phi(x)} f\left(n \varrho_{\nu}\right) \\
& =\sum_{\mu, \nu=1}^{\Phi(x)} f\left(\varrho_{\mu}-\varrho_{\nu}\right) .
\end{aligned}
$$

(iii) For $f(x)=\log |2 \sin \pi x|$, an even function in $\mathcal{K}_{1}$, we have

$$
-\sum_{\nu=1}^{\Phi(x)-1} \ell_{1}\left(\varrho_{\nu}\right)=\sum_{\nu=1}^{\Phi(x)-1} \log \left|2 \sin \pi \varrho_{\nu}\right|=\psi(x),
$$

where $\psi$ denotes Chebyshev's function. 
Proof. (i) Write $d=(m, n)$. Then by $\left(*_{s}\right)$ with $x=0$,

$$
\begin{aligned}
\sum_{k \bmod m} f\left(\frac{n k}{m}\right) & =\sum_{k \bmod m} f\left(\frac{(n / d) k}{m / d}\right)=d \sum_{k \bmod m / d} f\left(\frac{k}{m / d}\right) \\
& =d\left(\frac{m}{d}\right)^{1-s} f(0)=\frac{d^{s}}{m^{s-1}} f(0) .
\end{aligned}
$$

Now, by Lemma 3, the LHS of the formula in our proposition is

$$
\sum_{m \leq x} M\left(\frac{x}{m}\right) \sum_{k \bmod m} f\left(\frac{n k}{m}\right)
$$

and the assertion follows.

(ii) $\mathrm{By}\left(*_{s}\right)$

$$
f\left(n \varrho_{\nu}\right)=n^{s-1} \sum_{m=0}^{n-1} f\left(\varrho_{\nu}+\frac{m}{n}\right)=n^{s-1} \sum_{m=1}^{n} f\left(\varrho_{\nu}-\frac{m}{n}\right) .
$$

Hence

$$
\sum_{n \leq x} M\left(\frac{x}{n}\right) \frac{1}{n^{s-1}} f\left(n \varrho_{\nu}\right)=\sum_{\mu=1}^{\Phi(x)} f\left(\varrho_{\nu}-\varrho_{\mu}\right)
$$

in view of Lemma 3. Summing over $\nu$, the LHS [RHS] of this equality gives the leftmost [rightmost] member of the displayed formula, on using the formula in (i).

(iii) For $f(x)=\log |2 \sin \pi x|=\log \left|1-e^{2 \pi i x}\right|$ we have the modified form of $\left(*_{s}\right)$ :

$$
\sum_{k=1}^{m-1} f(k / m)=\log m
$$

This is formula (10) of Milnor [17], and is deduced trivially as there from the decomposition

$$
1+t+\ldots+t^{m-1}=\prod_{k=1}^{m-1}\left(t-e^{2 \pi i k / m}\right)
$$

(also follows from the Gauss multiplication formula for $\Gamma$ in logarithmic form and the reciprocity relation). Hence

$$
\sum_{\nu=1}^{\Phi(x)-1} \log \left|2 \sin \pi \varrho_{\nu}\right|=\sum_{m \leq x} M(x / m) \log m .
$$

Noting that

$$
\log m=\sum_{d \mid m} \Lambda(d)=\sum_{d \leq m} g(d / m)
$$


with

we infer from Lemma 3 that

$$
g\left(\frac{d}{m}\right)= \begin{cases}\Lambda(d), & d \mid m, \\ 0, & \text { otherwise },\end{cases}
$$

$$
\sum_{m \leq x} M\left(\frac{x}{m}\right) \log m=\sum_{\nu=1}^{\Phi(x)} g\left(n \varrho_{\nu}\right)=\sum_{m \leq x} \sum_{\substack{d=1 \\(d, m)=1}}^{m} g\left(\frac{m}{n}\right)=\sum_{n \leq x} \Lambda(n),
$$

thereby completing the proof of (iii).

Corollary. (i1) For $s \in \mathbb{N} \cup\{0\}$,

$$
\sum_{\nu=1}^{\Phi(x)} \bar{B}_{s}\left(n \varrho_{\nu}\right)=B_{s} \sum_{m \leq x} M\left(\frac{x}{m}\right) \frac{(m, n)^{s}}{m^{s-1}},
$$

and in particular,

(i2) For $\sigma>1$,

$$
\sum_{\nu=1}^{\Phi(x)} \bar{B}_{s}\left(\varrho_{\nu}\right)=B_{s} S_{s-1}(x)
$$

$$
\sum_{\nu=1}^{\Phi(x)} \ell_{s}\left(n \varrho_{\nu}\right)=\zeta(s) \sum_{m \leq x} M\left(\frac{x}{m}\right) \frac{(m, n)^{s}}{m^{s-1}}
$$

and in particular,

(ii)

$$
\sum_{\nu=1}^{\Phi(x)} \ell_{s}\left(\varrho_{\nu}\right)=\zeta(s) S_{s-1}(x)
$$

$$
\zeta(s) \sum_{m, n \leq x} M\left(\frac{x}{m}\right) M\left(\frac{x}{n}\right) \frac{(m, n)^{s}}{(m n)^{s-1}}=\sum_{\mu, \nu=1}^{\Phi(x)} \ell_{s}\left(\varrho_{\mu}-\varrho_{\nu}\right),
$$

and in particular,

$$
\begin{aligned}
\zeta(2)\left(12 \Phi(x) \sum_{\nu=1}^{\Phi(x)} \delta_{\nu}^{2}+1\right) & =\zeta(2) \sum_{m, n \leq x} M\left(\frac{x}{m}\right) M\left(\frac{x}{n}\right) \frac{(m, n)^{2}}{m n} \\
& =\sum_{\mu, \nu=1}^{\Phi(x)} \ell_{2}\left(\varrho_{\mu}-\varrho_{\nu}\right)=\pi^{2} \sum_{\mu, \nu=1}^{\Phi(x)} \bar{B}_{2}\left(\varrho_{\mu}-\varrho_{\nu}\right) .
\end{aligned}
$$

Theorem 2. (i) For $\operatorname{Re} s=\sigma \geq 3 / 2$, the estimate

$$
\sum_{\nu=1}^{\Phi(x)} \ell_{s}\left(\varrho_{\nu}\right)=O\left(x^{1 / 2+\varepsilon}\right)
$$

is equivalent to the $R H$. 
(ii) For $\sigma \geq 3 / 2$ and any $f \in \mathcal{K}_{s}$ that is not odd (and so at least for any even $f \in \mathcal{K}_{s}$ ) write its extension given in Lemma 6 again by $f$. Then the estimate

$$
\sum_{\nu=1}^{\Phi(x)} f\left(\varrho_{\nu}\right)=O\left(x^{1 / 2+\varepsilon}\right)
$$

is equivalent to the $R H$.

Pr o of. (i) is an immediate consequence of Corollary (i2) and Lemma 2.

(ii) We express $f \in \mathcal{K}_{s}$ as a linear combination of $\ell_{s}(x)$ and $\ell_{s}(1-x)$ :

$$
f(x)=c_{1} \ell_{s}(x)+c_{2} \ell_{s}(1-x) .
$$

Then the assumption amounts to $c_{1}+c_{2} \neq 0$. From Corollary (i2) it follows that

$$
\sum_{\nu=1}^{\Phi(x)} \ell_{s}\left(\varrho_{\nu}\right)=\sum_{\nu=1}^{\Phi(x)} \ell_{s}\left(1-\varrho_{\nu}\right)=\zeta(s) S_{s-1}(x)
$$

so that

$$
\sum_{\nu=1}^{\Phi(x)} f\left(\varrho_{\nu}\right)=\zeta(s)\left(c_{1}+c_{2}\right) S_{s-1}(x) .
$$

Now the result follows from Lemma 2.

COROllary 1. We have

$$
\sum_{\nu=1}^{\Phi(x)} \zeta_{1-s}\left(\varrho_{\nu}\right)=\zeta(1-s) S_{s-1}(x)
$$

so that for $\sigma \geq 3 / 2, s \neq 3,5, \ldots$ the estimate

$$
\sum_{\nu=1}^{\Phi(x)} \zeta_{1-s}\left(\varrho_{\nu}\right)=O\left(x^{1 / 2+\varepsilon}\right)
$$

is equivalent to the $R H$.

We follow Lewin [13] to define the Clausen functions:

$$
C l_{2 k}(\theta)=\sum_{n=1}^{\infty} \frac{\sin n \theta}{n^{2 k}}, \quad C l_{2 k+1}(\theta)=\sum_{n=1}^{\infty} \frac{\cos n \theta}{n^{2 k+1}} .
$$

Then, recursively,

$$
C l_{2 k+1}(\theta)=\zeta(2 k+1)-\int_{0}^{\theta} C l_{2 k}(\theta) d \theta, \quad C l_{2 k}(\theta)=\int_{0}^{\theta} C l_{2 k-1}(\theta) d \theta,
$$


and in particular we have the Clausen integral

$$
C l_{2}(\theta)=-\int_{0}^{\theta} \log |2 \sin (\theta / 2)| d \theta \quad\left(C l_{1}(\theta)=-\log |2 \sin (\theta / 2)|\right) .
$$

For $k \in \mathbb{N}$ we have the Fourier expansion

$$
\begin{aligned}
\ell_{k}(x) & =\sum_{n=1}^{\infty} \frac{\cos 2 \pi n x}{n^{k}}+i \sum_{n=1}^{\infty} \frac{\sin 2 \pi n x}{n^{k}} \\
& =\frac{(2 \pi i)^{k-1}}{k !}\left[\frac{(-1)^{(k-1) / 2} k !}{(2 \pi)^{k-1}} C l_{k}(2 \pi x)-\pi i \bar{B}_{k}(x)\right] .
\end{aligned}
$$

Corollary 2 (cf. Milnor's table [17]). For each $k \in \mathbb{N}$ each of the following is equivalent to the $R H$ :

$$
\sum_{\nu=1}^{\Phi(x)} \bar{B}_{2 k}\left(\varrho_{\nu}\right)=O\left(x^{1 / 2+\varepsilon}\right), \quad \sum_{\nu=1}^{\Phi(x)} C l_{2 k+1}\left(\varrho_{\nu}\right)=O\left(x^{1 / 2+\varepsilon}\right) .
$$

Remark 5. Milnor uses the notation $\Lambda(\pi x)=-\int_{0}^{\pi x} \log |2 \sin \theta| d \theta$, which is $\frac{1}{2} C l_{2}(2 \pi x)$. Also, in Yamamoto's notation [20], $A_{k}(x)$ is related to $C l_{k}(x)$ by

$$
A_{k}(x)=\frac{(-1)^{(k-1) / 2} k !}{(2 \pi)^{k-1}} C l_{k}(2 \pi x) .
$$

Proposition 4. We have

$$
\sum_{\varrho_{\nu}<1 / 2} \log \tan \pi \varrho_{\nu}=\frac{1}{2}\left(\psi(x)-\psi\left(\frac{x}{2}\right)\right)-\frac{1}{2} \log 2,
$$

and so the estimate

$$
\sum_{\varrho_{\nu}<1 / 2} \log \tan \pi \varrho_{\nu}-x / 4=O\left(x^{1 / 2+\varepsilon}\right)
$$

is equivalent to the RH. Similar formulas hold for cotangents.

Pr o of. From the well-known formulas

$$
\prod_{r=1}^{n-1} \tan \frac{r}{2 n} \pi=1, \quad \prod_{r=1}^{n} \tan \frac{r}{2 n+1} \pi=\sqrt{2 n+1},
$$

we deduce that

$$
\sum_{r<n / 2} \log \tan \frac{r}{n} \pi= \begin{cases}\frac{1}{2} \log n, & 2 \nmid n, \\ 0, & 2 \mid n .\end{cases}
$$


Hence by Lemma 3,

$$
\begin{aligned}
\sum_{\varrho_{\nu}<1 / 2} \log \tan \pi \varrho_{\nu} & =\sum_{n \leq x} M\left(\frac{x}{n}\right) \frac{1}{2} \log n-\sum_{n \leq x} M\left(\frac{x}{2 n}\right) \frac{1}{2} \log 2 n \\
& =\frac{1}{2} \sum_{x / 2<n \leq x} M\left(\frac{x}{n}\right) \log n-\frac{1}{2} \log 2 \\
& =\frac{1}{2}\left(\psi(x)-\psi\left(\frac{x}{2}\right)\right)-\frac{1}{2} \log 2,
\end{aligned}
$$

thereby completing the proof of Lemma 4 and (2). For the cotangent function we have

$$
\sum_{\varrho_{\nu}<1 / 2} \log \cot \pi \varrho_{\nu}=-\frac{1}{2}\left(\psi(x)-\psi\left(\frac{x}{2}\right)\right)+\frac{1}{2} \log 2 .
$$

6. Franel's theorem. In this section we will show that Franel's celebrated formula has its origin in the three-term relation for the generalized Dedekind sums (Lemma 8).

LEMMA 7.

$$
\sum_{\nu=1}^{\Phi(x)} \bar{B}_{1}\left(a \varrho_{\nu}\right) \bar{B}_{1}\left(b \varrho_{\nu}\right)=\sum_{c \leq x} M\left(\frac{x}{c}\right) \sum_{k=1}^{c} \bar{B}_{1}\left(\frac{a k}{c}\right) \bar{B}_{1}\left(\frac{b k}{c}\right) .
$$

Proof. This follows from Lemma 3 with $\xi=1$ and $f(t)=\bar{B}_{1}(a t) \bar{B}_{1}(b t)$ :

$$
\text { LHS }=\sum_{c \leq x} M\left(\frac{x}{c}\right) V_{1}(c)=\sum_{c \leq x} M\left(\frac{x}{c}\right) \sum_{k \leq c} f\left(\frac{k}{c}\right) .
$$

Lemma 8 (The three-term relation). For any natural numbers $a, b, c$ let

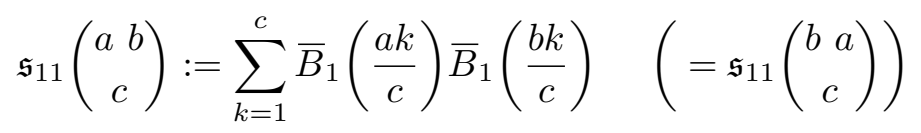

denote a modification of Mikolás's generalized Dedekind sum. Then

$$
\begin{aligned}
& \mathfrak{s}_{11}\left(\begin{array}{cc}
a & b \\
c
\end{array}\right)+\mathfrak{s}_{11}\left(\begin{array}{cc}
b & c \\
a
\end{array}\right)+\mathfrak{s}_{11}\left(\begin{array}{cc}
c & a \\
b
\end{array}\right) \\
& =\frac{1}{12}\left(\frac{(a, b)^{2}}{a b} c+\frac{(b, c)^{2}}{b c} a+\frac{(c, a)^{2}}{c a} b\right)+\frac{(a, b, c)}{2} .
\end{aligned}
$$

Proof. The proof closely follows the pattern of that of Sczech's Satz 1 [19] and we will state analogues of his results that are needed for the proof. 
The analogue of formula (9): For real $x, y, z$ with $x+y+z=0$,

$$
\begin{array}{r}
2\left\{\bar{B}_{1}(x) \bar{B}_{1}(y)+\bar{B}_{1}(y) \bar{B}_{1}(z)+\bar{B}_{1}(z) \bar{B}_{1}(x)\right\}+\bar{B}_{2}(x)+\bar{B}_{2}(y)+\bar{B}_{2}(z) \\
= \begin{cases}2, & x, y \in \mathbb{Z}, \\
0, & \text { otherwise. }\end{cases}
\end{array}
$$

Then we put $x=u_{1}-u_{2}, y=u_{3}-u_{1}, z=u_{2}-u_{3}$.

The analogue of formula (10): For real independent variables $u_{j}$, $j \bmod 3$,

$$
\begin{aligned}
\sum_{j \bmod 3} \bar{B}_{2}\left(u_{j}-u_{j+1}\right)-2 \sum_{j \bmod 3} \bar{B}_{1}\left(u_{j}-u_{j+1}\right) \bar{B}_{1}\left(u_{j}-u_{j-1}\right) \\
= \begin{cases}0, & x, y, z \in \mathbb{R}-\mathbb{Z}, \\
-1, & x, y, z \in \mathbb{Z}, \\
2 \bar{B}_{1}(y), & z \in \mathbb{Z}, x, y \in \mathbb{R}-\mathbb{Z} .\end{cases}
\end{aligned}
$$

Put $u_{j}=\left(r_{j}+z_{j}\right) / c_{j}, r_{j} \bmod 3, z_{j} \in \mathbb{R}$. Then the analogue of the first formula on p. 530 of [19] (with $z=(0,0,0)$ ) is

$$
\sum_{\substack{r_{l}\left(c_{l}\right) \\ l=1,2,3}} \bar{B}_{2}\left(\frac{r_{j}}{c_{j}}-\frac{r_{j+1}}{c_{j+1}}\right)=\frac{\left(c_{j}, c_{j+1}\right)^{2}}{6 c_{j} c_{j+1}} c_{j-1},
$$

while the second formula on p. 530 (with $z=(0,0,0)$ ) is

$$
\sum_{\substack{r_{l}\left(c_{l}\right) \\
l=1,2,3}} \bar{B}_{1}\left(u_{j}-\frac{r_{j+1}}{c_{j+1}}\right) \bar{B}_{1}\left(u_{j}-\frac{r_{j-1}}{c_{j-1}}\right)=\mathfrak{s}\left(\begin{array}{c}
c_{j+1} c_{j-1} \\
c_{j}
\end{array}\right) .
$$

Thus the analogue of Satz 1 (with $z=(0,0,0)$ ) is

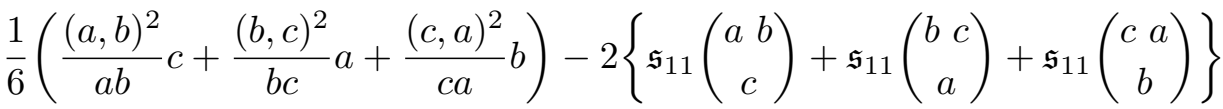

$$
\begin{aligned}
& =\sum_{\substack{r_{l}\left(c_{l}\right) \\
l=1,2,3}}\left\{\begin{array}{l}
0, \\
-1, \\
2 \bar{B}_{1}(y),
\end{array}\right.
\end{aligned}
$$

where on the RHS the same conditions as in $\left(10^{\prime}\right)$ should apply.

We now digress slightly from Sczech and calculate the RHS. Considering the summation condition, we have by the distribution property

$$
\sum_{\substack{r_{l}\left(c_{l}\right), l=1,2,3 \\ z \in \mathbb{Z}}} \bar{B}_{1}\left(\frac{r_{3}}{c_{3}}-\frac{r_{1}}{c_{1}}\right)=-\frac{1}{2}\left(c_{1}, c_{2}, c_{3}\right) .
$$


Further,

$$
\begin{aligned}
\sum_{\substack{r_{l}\left(c_{l}\right), l=1,2,3 \\
x, y \in \mathbb{R}-\mathbb{Z}, z \in \mathbb{Z}}} \bar{B}_{1}\left(\frac{r_{3}}{c_{3}}-\frac{r_{1}}{c_{1}}\right) \\
=\sum_{\substack{r_{l}\left(c_{l}\right), l=1,2,3 \\
z \in \mathbb{Z}}} \bar{B}_{1}\left(\frac{r_{3}}{c_{3}}-\frac{r_{1}}{c_{1}}\right)-\sum_{\substack{r_{l}\left(c_{l}\right), l=1,2,3 \\
x, y, z \in \mathbb{Z}}} \bar{B}_{1}\left(\frac{r_{3}}{c_{3}}-\frac{r_{1}}{c_{1}}\right) \\
=-\frac{1}{2}\left(c_{1}, c_{2}, c_{3}\right)+\frac{1}{2}\left(c_{1}, c_{2}, c_{3}\right)=0
\end{aligned}
$$

since

$$
\sum_{\substack{r_{l}\left(c_{l}\right), l=1,2,3 \\ x, y, z \in \mathbb{Z}}} \bar{B}_{1}\left(\frac{r_{3}}{c_{3}}-\frac{r_{1}}{c_{1}}\right)=B_{1} \sum_{h \bmod \left(c_{1}, c_{2}, c_{3}\right)} 1=-\frac{1}{2}\left(c_{1}, c_{2}, c_{3}\right),
$$

which shows incidentally that the sum $\sum_{r_{l}\left(c_{l}\right), l=1,2,3, x, y, z \in \mathbb{Z}}(-1)$ is $-\left(c_{1}, c_{2}, c_{3}\right)$. This completes the proof.

Theorem 3 (Franel's formula). We have the identity

$$
\sum_{\nu=1}^{\Phi(x)} \delta_{\nu}^{2}=\frac{1}{12 \Phi(x)}\left(\sum_{m, n \leq x} M\left(\frac{x}{m}\right) M\left(\frac{x}{n}\right) \frac{(m, n)^{2}}{m n}-1\right) .
$$

Proof. First note that by Proposition 1 we have

$$
\delta_{\nu}=\varrho_{\nu}-\frac{\nu}{\Phi(x)}=\varrho_{\nu}-\frac{1}{\Phi(x)} h\left(\varrho_{\nu}\right)=\frac{1}{\Phi(x)}\left(\sum_{n \leq x} M\left(\frac{x}{n}\right) \bar{B}_{1}\left(n \varrho_{\nu}\right)+\frac{1}{2}\right),
$$

whence it follows that

$$
\begin{aligned}
\sum_{\nu=1}^{\Phi(x)} \delta_{\nu}^{2}= & \frac{1}{\Phi(x)^{2}} \sum_{a \leq x} \sum_{b \leq x} M\left(\frac{x}{a}\right) M\left(\frac{x}{b}\right) \sum_{\nu=1}^{\Phi(x)} \bar{B}_{1}\left(a \varrho_{\nu}\right) \bar{B}_{1}\left(b \varrho_{\nu}\right) \\
& +\frac{1}{\Phi(x)^{2}} \sum_{n \leq x} M\left(\frac{x}{n}\right) \sum_{\nu=1}^{\Phi(x)} \bar{B}_{1}\left(n \varrho_{\nu}\right)+\frac{1}{4 \Phi(x)} .
\end{aligned}
$$

Since by Corollary 1(i) to Proposition 3, the second term becomes

$$
-\frac{1}{2 \Phi(x)^{2}} \sum_{m, n \leq x} M\left(\frac{x}{m}\right) M\left(\frac{x}{n}\right)(m, n),
$$

the main ingredient is the first term, which can be transformed further using Lemma 7 as follows:

$$
\sum_{a \leq x} \sum_{b \leq x} M\left(\frac{x}{a}\right) M\left(\frac{x}{b}\right) \sum_{\nu=1}^{\Phi(x)} \bar{B}_{1}\left(a \varrho_{\nu}\right) \bar{B}_{1}\left(b \varrho_{\nu}\right)
$$




$$
\begin{aligned}
& =\sum_{a \leq x} \sum_{b \leq x} M\left(\frac{x}{a}\right) M\left(\frac{x}{b}\right) \sum_{c \leq x} M\left(\frac{x}{c}\right) \sum_{k=1}^{c} \bar{B}_{1}\left(\frac{a k}{c}\right) \bar{B}_{1}\left(\frac{b k}{c}\right) \\
& =\sum_{a, b, c \leq x} M\left(\frac{x}{a}\right) M\left(\frac{x}{b}\right) M\left(\frac{x}{c}\right) \mathfrak{s}_{11}\left(\begin{array}{c}
a b \\
c
\end{array}\right),
\end{aligned}
$$

where $\mathfrak{s}_{11}\left(\begin{array}{c}a b \\ c\end{array}\right)$ denotes a modification of Mikolás's generalized Dedekind sum.

Put

$$
S(x):=\sum_{a, b, c \leq x} M\left(\frac{x}{a}\right) M\left(\frac{x}{b}\right) M\left(\frac{x}{c}\right) \mathfrak{s}_{11}\left(\begin{array}{c}
a b \\
c
\end{array}\right) .
$$

Then also

$$
\begin{aligned}
& S(x)=\sum_{a, b, c \leq x} M\left(\frac{x}{a}\right) M\left(\frac{x}{b}\right) M\left(\frac{x}{c}\right) \mathfrak{s}_{11}\left(\begin{array}{c}
b c \\
a
\end{array}\right)
\end{aligned}
$$

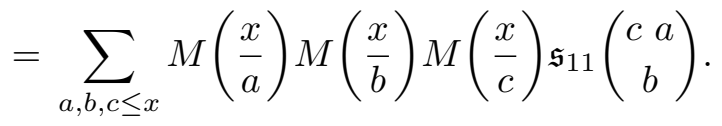

Hence

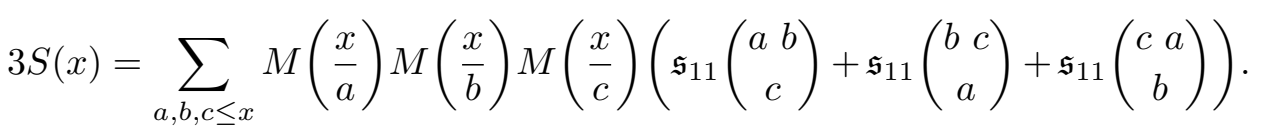

Substituting this in the above formula, we infer that

$$
\begin{aligned}
3 \Phi(x)^{2} \sum_{\nu=1}^{\Phi(x)} \delta_{\nu}^{2}= & \frac{1}{12} \sum_{a, b, c \leq x} M\left(\frac{x}{a}\right) M\left(\frac{x}{b}\right) M\left(\frac{x}{c}\right) \\
& \times\left\{\frac{(a, b)^{2}}{a b} c+\frac{(b, c)^{2}}{b c} a+\frac{(c, a)^{2}}{c a} b\right\} \\
& +\frac{1}{2} \sum_{a, b, c \leq x} M\left(\frac{x}{a}\right) M\left(\frac{x}{b}\right) M\left(\frac{x}{c}\right)(a, b, c) \\
& -\frac{3}{2} \sum_{m, n \leq x} M\left(\frac{x}{m}\right) M\left(\frac{x}{n}\right)(m, n)+\frac{3}{4} \Phi(x) .
\end{aligned}
$$

Noting that the first term on the LHS becomes

$$
\frac{\Phi(x)}{4} \sum_{m, n \leq x} M\left(\frac{x}{m}\right) M\left(\frac{x}{n}\right) \frac{(m, n)^{2}}{m n},
$$


while the second reduces to

$$
\begin{aligned}
\sum_{d \leq x} d & \sum_{\substack{a, b, c \leq x / d \\
(a, b, c)=1}} M\left(\frac{x}{d a}\right) M\left(\frac{x}{d b}\right) M\left(\frac{x}{d c}\right) \\
= & \sum_{d \leq x} d \sum_{a, b \leq x / d} M\left(\frac{x}{d a}\right) M\left(\frac{x}{d b}\right) \sum_{\delta \mid(a, b)} \mu(\delta) \sum_{c^{\prime} \leq x /(d \delta)} M\left(\frac{x}{d \delta c^{\prime}}\right) \\
= & \sum_{d \leq x} d \sum_{a \leq x / d} M\left(\frac{x}{d a}\right) \sum_{\delta \mid a} \mu(\delta) \sum_{b^{\prime} \leq x /(d \delta)} M\left(\frac{x}{d \delta b^{\prime}}\right) \\
= & \sum_{d \leq x} d M\left(\frac{x}{d}\right)=\Phi(x),
\end{aligned}
$$

which in passing also shows that the third term is again $\Phi(x)$, we complete the proof.

\section{References}

[1] P. Codecà, Alcune proprietà della discrepanza locale delle sequenze di Farey, Atti Accad. Sci. Istit. Bologna 13 (1981), 163-173.

[2] P. Codecà and A. Perelli, On the uniform distribution $(\bmod 1)$ of the Farey fractions and $\ell^{p}$ spaces, Math. Ann. 279 (1988), 413-422.

[3] J. Franel, Les suites de Farey et les problèmes des nombres premiers, Nachr. Ges. Wiss. Göttingen Math.-Phys. Kl. 1924, 198-201.

[4] A. Fujii, A remark on the Riemann hypothesis, Comment. Math. Univ. St. Paul. 29 (1980), 195-201.

[5] - Some explicit formulas in number theory, Proc. Japan Acad. Ser. A Math. Sci. 57 (1981), 326-330.

[6] M. N. Huxley, The distribution of Farey points, I, Acta Arith. 18 (1971), 281-287.

[7] M. Ishibashi and S. Kanemitsu, Fractional part sums and divisor functions I, in: Number Theory and Combinatorics, J. Akiyama et al. (eds.), World Sci., 1985, 119-193.

[8] T. Kano, The Riemann Hypothesis, Nihonhyôronsha, 1990 (in Japanese).

[9] J. Kopriva, On a relation of the Farey series to the Riemann hypothesis on the zeros of the $\zeta$ function, Casopis Pěst. Mat. 78 (1953), 49-55 (in Czech).

[10] - Contribution to the relation of the Farey series to the Riemann hypothesis, ibid. 79 (1954), 77-82 (in Czech).

[11] E. Landau, Bemerkungen zu vorstehenden Abhandlung von Herrn Franel, Nachr. Ges. Wiss. Göttingen Math.-Phys. Kl. 1924, 202-206; Collected Works, Vol. 8, Thales Verlag.

[12] —, Vorlesungen über Zahlentheorie, Teubner, 1927; Chelsea reprint, 1947.

[13] L. Lewin, Polylogarithms and Associated Functions, North-Holland, 1981.

[14] M. Mikolás, Farey series and their connection with the prime number problem I, Acta Sci. Math. (Szeged) 13 (1949), 93-117. 
[15] M. Mikolás, Farey series and their connection with the prime number problem II, ibid. 14 (1951), 5-21.

[16] M. Mikolás and K.-I. Sato, On the asymptotic behaviour of Franel's sum and the Riemann hypothesis, Results Math. 21 (1992), 368-378.

[17] J. Milnor, On polylogarithms, Hurwitz zeta functions, and the Kubert identities, Enseign. Math. 29 (1983), 281-322.

[18] H. Niederreiter, The distribution of Farey points, Math. Ann. 201 (1973), 341345 .

[19] R. Sczech, Dedekindsummen mit elliptischen Funktionen, Invent. Math. 76 (1984), $523-551$

[20] Y. Y amamoto, Dirichlet series with periodic coefficients, in: Proc. Intern. Sympos. Algebraic Number Theory, Kyoto 1976, Japan Society for the Promotion of Science, 1977, 275-289.

[21] A. Zulauf, The distribution of Farey numbers, J. Reine Angew. Math. 289 (1977), 209-213.

S. Kanemitsu

Department of Liberal Arts and Sciences

University of Kinki, Iizuka

Fukuoka 820, Japan
M. Yoshimoto

Department of Mathematical Sciences Faculty of Science Kyushu University Fukuoka 812, Japan 